\title{
Prevalence of Helicobacter pylori and Determination of Antibiotic Resistance in Patients with Gastritis Referred to Shahid Beheshti University of Medical Sciences Hospitals in Tehran Between 2010 and 2011
}

\author{
Gita Eslami ${ }^{1, *}$, Soudabeh Taheri ${ }^{1}$, Neda Baseri ${ }^{2}$, Seyed Ali Montazeri ${ }^{2}$, Abdolvahid Shakeri ${ }^{2}$, \\ Roghayeh Samadi ${ }^{2}$, Hosein Dabiri $^{1}$, Zahra Zahirnia ${ }^{2}$, Azanollah Azargashb ${ }^{3}$ \\ ${ }^{1}$ Department of Microbiology, Faculty of Medicine, Shahid Beheshti University of Medical Sciences, Tehran, IR Iran \\ ${ }^{2}$ Faculty of Medicine, Shahid Beheshti University of Medical Sciences, Tehran, IR Iran \\ ${ }^{3}$ Department of Community Medicine and Health, Faculty of Medicine, Shahid Beheshti University of Medical Sciences, Tehran, IR Iran \\ *Corresponding author: Gita Eslami, Faculty of Medicine, Shahid Beheshti University of Medical Sciences, Tehran, IR Iran. Tel.: +98-9123729180, Fax:+98-2122439964, E-mail: g_eslami@ \\ yahoo.com.
}

Received: September 17, 2012; Revised: October 25, 2012; Accepted: November 29, 2012

\begin{abstract}
Background: Prevalence of Helicobacter pylori (H. pylori) differs among populations worldwide. H. pylori has been correlated with gastritis, peptic ulcer and cancer. Considering the treatment failure due to antibiotic resistance, proper treatment of $H$. pylori infection is of great importance.

Objectives: The aim of this study was to determine the prevalence of H. pylori infection based on age, sex and also to examine antibiotic resistance among patients referred to the endoscopy department of Shahid Beheshti University of Medical Sciences hospitals in Tehran from 2010 to 2011.

Patients and Methods: In this descriptive study, biopsies were taken from 192 patients with dyspepsia who underwent endoscopic evaluation. For diagnosis of $H$. pylori infection and histologic assessment, samples were stained by Giemsa and hematoxylin-eosin (H \& E) staining. Based on the Sydney system for classification of gastritis, extent of $H$. pylori infection and severity of gastritis were measured. To determine antibiotic resistance after culturing on Brucella agar medium, antibiogram test was done using modified disk diffusion method on Mueller-Hinton agar considering standard conditions. Data analysis was done by the SPSS 16.0 software, using the chi-square and T-test methods.

Results: Overall prevalence of $\mathrm{H}$. pylori was $83 \%$ among all patients and $90.3 \%$ among those with gastritis $(\mathrm{CI} 95 \%=85.9 \%-94.6 \%)$. There was no significant correlation between the extent of $H$. pylori infection and age $(\mathrm{P}=0.182)$ and gender $(\mathrm{P}=0.466)$. Yet, there was a significant correlation between $H$. pylori infection and severity of gastritis $(\mathrm{P}=0.001)$. Rates of resistance to metronidazole, clarithromycin, amoxicillin, ciprofloxacin and tetracycline were $50 \%, 16.1 \%, 5.2 \%, 4.6 \%$ and $3.6 \%$, respectively.

Conclusions: Prevalence of $\mathrm{H}$. pylori infection in our patients was similar to that of developing countries. Severity of gastritis is correlated with the extent of $H$. pylori infection. Moreover, the antibiotic resistance rate observed in this study signifies the ever-growing importance of further antibiotic sensitivity studies to help with proper treatment regimens against $H$. pylori.
\end{abstract}

Keywords: Prevalence; Helicobacter pylori; Gastritis; Iran

\section{Background}

In 1983, presence of a gram-negative, spiral and microaerophilic bacterium in patients with gastritis and peptic ulcer was reported by three different research groups in the literature. This spiral bacterium was first called "Campylobacter-like organism" but after a while it was renamed as Helicobacter pylori (H. pylori) (1). H. pylori is usually present either in the deep layers of lining mucosa of gastric epithelium or between those layers and gastric epithelium (2). This organism is adapted for colonization in the human stomach and exists in almost half of the human population, but different species of $H$. pylori cause increased risk of diseases like gastritis and thereinafter gastric ulcer, duodenal ulcer, gastric cancer and mucosal associated lymphoid tissue (MALT) lymphoma by their pathogenic factors such as urease, BabA, OipA, CagPA1, SabA and $\operatorname{VacA}(3,4)$.

The term "gastritis" is used when inflammation of the gastric mucosal layer is confirmed histologically. This disease can be categorized based on the duration (acute or chronic gastritis), histologic features, anatomical distribution and possible mechanisms of pathogenecity. At present, it is believed that any factor causing primary le- 
sion in gastric mucosal layer can lead to gastritis and gastric atrophy (in chronic conditions), which is considered an important risk factor for gastric cancer (5). There are wide and heterogenous factors for incidence of gastritis including biliary reflux into the stomach, non-steroidal anti-inflammatory drugs (NSAIDs), stress, inappropriate diet, cigarette smoking, severe anemia and immune system disorders. Many previous studies have been conducted to determine the prevalence of $H$. pylori in the general population and patients with upper gastrointestinal problems including gastritis. Based on their results, prevalence of $H$. pylori differs among populations in the world and is strongly correlated with life standards like socio-economic status, educational level, and health condition $(6,7)$. Prevalence of $H$. pylori infection in Iranian adults was reported at up to $80 \%$ based on serological studies (8).

H. pylori eradication is of clinical importance to decrease gastric inflammatory diseases and to prevent progression towards carcinoma. Multi-drug regimens have been proposed as the most effective therapy. Examples are: 1) three-drug regimen: omeprazole, clarithromycin, amoxicillin or metronidazole; 2) four-drug regimen: a bismuth derivative, metronidazole, tetracycline and a proton-pump inhibitor. One of the most important reasons for H. pylori treatment failure is the ever-growing antibiotic resistance resulting in lack of treatment efficacy particularly in developing countries (9-11).

\section{Objectives}

Regarding the importance of H. pylori infection and resistance to current regimens, this study was conducted to determine the prevalence of $H$. pylori infection in patients with gastritis, referred to Shahid Beheshti University of Medical Sciences Hospitals in Tehran from 2010 to 2011, based on gender, age and the level of antibiotic resistance. The use of this information can be of value to consider appropriate treatment regimens.

\section{Patients and Methods}

In this descriptive study, 192 patients with dyspepsia who presented clinical symptoms of abdominal pain, flatus, heart-burn, nausea and vomiting, after being examined by a gastroenterologist, underwent upper endoscopy. Informed consent was obtained and those who volunteered were asked to fill a questionnaire including age, sex, and drug history. Excluding criteria in the study were previous use of antacids (e.g. Omeprazole, Bismuth) and antibiotics. To perform an endoscopy, all of the patients were first prepared with the help of ward staff, and finally two samples of gastric antrum were biopsied by the gastroenterologist. Histological assessment:samples were fixed in $10 \%$ formaldehyde solution, then put into paraffin under the biological hood and cut into four-micrometer slices by a sterile pincet. For histological assessment and H. pylori diagnosis, hematoxylin-eosin (H \& E) and Giemsa stains were used. In this study, the extent of H. pylori infection and severity of gastritis for all the samples were measured based on the latest version of Sydney system for the classification of gastritis and categorized into "mild and moderate" and "severe" (12). Moreover, association between $H$. pylori and severity of gastritis was assessed.

Culturing of the samples and antibiogram test to determine antibiotic resistance: biopsy slices were cultured on Brucella agar medium (Merck Co., Germany) containing $10 \%$ sheep blood, vancomycin $(6 \mathrm{mg} / \mathrm{L})$, trimethoprim $(5$ $\mathrm{mg} / \mathrm{L})$, and amphotericin $\mathrm{B}(2 \mathrm{mg} / \mathrm{L})$. The cultured plates were incubated in a micro-aerobic atmosphere $\left(5 \% \mathrm{O}_{2}, 10 \%\right.$ $\mathrm{CO}_{2}$, and $85 \% \mathrm{~N}_{2}$ ) of a $\mathrm{CO}_{2}$ incubator (Innova Co-170; New Brunswick Scientific, Edison, NJ, USA) at $37^{\circ} \mathrm{C}$ for three to five days. Grown colonies were identified using urease, catalase and oxidase tests as well as Gram staining $(8,13)$. Next, to determine antibiotic sensitivity, an antibiogram test was done using the modified disk diffusion method. At this stage, $100 \mu \mathrm{L}$ bacterial suspension was as prepared equivalent to the No. 3 McFarland standard and cultured on Mueller-Hinton agar medium (Merck Co., Germany) containing $5 \%$ defibrinated sheep blood. Finally, antibiotic disks were placed on the medium and stored in a $\mathrm{CO}_{2}$ incubator in microaerophilic conditions for three to five days. Following the incubation, antimicrobial effects of antibiotics were categorized into sensitive and resistant by measuring the inhibition zone diameters (based on CLSI standards) in millimeters (14). It should be mentioned that in this study, semi-sensitive status was not considered. Antibiotic disks used were tetracycline ( $\mathrm{Pa}-$ tan teb.), amoxicillin (Patan teb.), metronidazole (MAST, London), ciprofloxacin (Patan teb.) and clarithromycin (MAST, London). Statistical analysis: data was analyzed using the SPSS software (version 16.0). For categorical variables the chi-square test and for continuous variables the T-test was used.

\section{Results}

Of the 192 patients, 85 were female (45\%) and 107 were male (55\%). The youngest was a 16-year-old female and the oldest was an 88-year-old female. Mean $\pm \mathrm{SD}$ age of the patients was $48.9 \pm 16.9$ years. The frequency of $H$. pylori infection was $83 \%$. In those younger than 20 years of age, the frequency was lower than that of the older group and despite observing more positive test results in the older age groups, this was not statistically significant $(\mathrm{P}$ value $=$ 0.182 ) (Table 1).

Frequency of $H$. pylori infection in female subjects was $80 \%$ and in males was $85.1 \%$. Considering the severity of $H$. pylori infection, in the "severe" category, 35 subjects were male $(71 \%)$ and 17 subjects $(29 \%)$ were female $(P=0.056)$. However, no statistically significant correlation was found between sex and severity of infection $(P=0.466)$ (Table 2$)$. 
Eslami G et al.

Table 1. Frequency and Percentage of H. pylori Infection in Patients Referred to Shahid Beheshti University of Medical Sciences Hospitals in Tehran from 2010 to 2011 (Age-Wise)

\begin{tabular}{llll}
\hline Age & Helicobacter pylori, Negative, No. (\%) & Helicobacter pylori, Positive, No. (\%) & Total Patients \\
\hline 0- 20 & $2(50)$ & $2(50)$ & 4 \\
\hline $20-40$ & $8(13)$ & $54(87)$ & 62 \\
\hline $\mathbf{4 0 - 6 0}$ & $10(15.2)$ & $56(84.8)$ & 66 \\
$\geq \mathbf{6 0}$ & $13(21.6)$ & $47(78.4)$ & 60 \\
Total & $33(17)$ & $159(83)$ & 192 \\
\hline
\end{tabular}

Table 2. Frequency and Percentage of H.pylori Infection in Patients Referred to Shahid Beheshti University of Medical Sciences Hospitals in Tehran from 2010 to 2011 (Gender-Wise)

\begin{tabular}{llll}
\hline Gender & Helicobacter pylori, Negative, No. (\%) & Helicobacter pylori, Positive, No. (\%) & Total Patients \\
\hline Male & $16(14.9)$ & $91(85.1)$ & 107 \\
Female & $17(20)$ & $68(80)$ & 85 \\
\hline
\end{tabular}

In this survey, 176 of 192 patients had gastritis. Frequency of $H$. pylori in these 176 cases was $90.3 \%$ (CI 95\%= 85.9\% 94.6\%). There was a significant correlation between severity of gastritis and $H$. pylori infection. Of the 53 subjects with severe $H$. pylori infection, 35 patients also had severe gastritis (66.1\%), while among subjects with mild to moderate $H$. pylori infection only 13 (12\%) had severe gastritis (Figure 1).

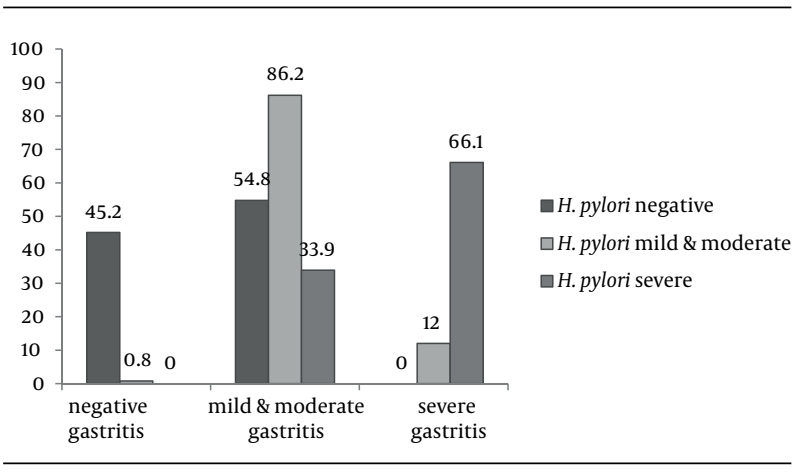

Figure 1. Correlation of Helicobacter pylori (H. pylori) Infection and Severity of Gastritis (Percentages) for Patients Referred to Shahid Beheshti University of Medical Sciences Hospitals in Tehran From 2010 to 2011

The resistance status of isolated $H$. pylori samples from patients were as follows: resistance rates to metronidazole, clarithromycin, amoxicillin, ciprofloxacin and tetracycline clarithromycin, amoxicillin, ciprofloxacin and tetracycline were $50 \%, 16.1 \%, 5.2 \%, 4.6 \%$ and $3.6 \%$, respectively.

\section{Discussion}

Based on the results of this study, the frequency of $H$. pylori infection in our patients was $83 \%$. This finding is in accordance with previous studies conducted in developing countries, reported that the rate of prevalence for $H$. pylori infection was approximately $75 \%$, while studies in the developed countries have shown a much lower preva- lence rate, which was around 40\% (15-17). Considering the fact that in this study subjects had dyspepsia, a higher frequency would be expected. Although some studies have suggested an increasing rate of $H$. pylori infection with age (18), this study did not find such association. In this study, children were not included, so no comparison could be made with studies investigating this age group. In 2007, Sasidharan et al. showed a significantly higher frequency for $H$. pylori infection among males, compared to females (51.9\% versus 33.1\%) (19). According to many studies, differences observed between genders might be due to different life styles such as cigarette smoking and alcohol consumption, which in males can be a predisposing factor for $H$. pylori infection (20). Furthermore, iron deficiency anemia in women as a result of menstruation can decrease the frequency of $H$. pylori infection (21). In contrast, some researchers believe H. pylori infection dose not correlate with gender (22). We showed that $71 \%$ of severely infected subjects were males, while only $29 \%$ were females. However, overall analysis failed to show any significant association; infection in men was only 5\% higher than women.

Like many other studies, this study showed that $H$. pylori infection is associated with gastritis $(23,24)$. We observed a significant correlation between $H$. pylori virulence and severity of gastritis. $H$. pylori had the strongest correlation with "severe gastritis". Among patients with severe H. pylori infection, $66.1 \%$ had severe gastritis. A study in Jordan revealed that about $82 \%$ of dyspeptic patients, most of whom had gastritis, were infected with $H$. pylori (25). In 2003, another study showed that prevalence of $H$. pylori infection in patients with upper gastrointestinal disorders like gastritis was $68.5 \%$. This result is suggestive of a strong correlation between $H$. pylori infection and development of gastritis in different populations (26). With an assumption that there is high prevalence of $H$. pylori infection in our country, high risk patients should be treated to prevent development of gastric cancer. More- 
over, specific analysis to detect drug resistance must be undertaken to prevent treatment failure of $H$. pylori infection. In samples assessed in this study, resistance to metronidazole was considerably high (50\%), which corresponds with the results of the Siavoshi et al. (55.6\%) (27) and Mohammadi et al. studies from Tehran (52\%) (8). It seems that widespread use of metronidazole in $H$. pylori and parasitic infections has resulted in the emergence of highly resistant species to this antibiotic $(28,29)$.

In this study, resistance rate to clarithromycin was $16.1 \%$ and this is similar to the rates reported by Rafeey et al. (30) and Mohammadi et al. (8), which were $16 \%$ and $17 \%$, respectively. Other studies reported 7.3\% (27) and 30.1\% (31) as the rate of resistance to clarithromycin $(27,31)$. In this study, rate of resistance to amoxicillin was $5.2 \%$, which was similar to that of one study from Tehran performed during 2010 (7.3\%) (27). However, it was lower than the results of studies from Kerman (27\%) and Shiraz (20.8\%) $(31,32)$. Rate of resistance to ciprofloxacin in this study (4.6\%) was similar to those reported from Tehran, during 2007 (30) and Kerman, during 2009 (31), which were 7\% and $7.9 \%$, respectively. Rate of resistance to tetracycline in this study (3.6\%) was to some extent similar to other studies $(31,32)$. In this study, rate of resistance to furazalidone was not determined. However, regarding the limited number of studies, which have investigated resistance to this drug, an increasing rate of resistance is suggested. In a study by Siavoshi et al. in 2000 (33), this rate was $0 \%$ while in 2010 (27) it was $4.5 \%$.

Studies worldwide, have reported different antibiotic resistance rates. Boyanova et al. in 2010 showed a high resistance rate to clarithromycin (over 20\%) in the US, developed countries, Europe and Asia. The highest resistance rate to metronidazole (over $80 \%$ ) was reported for Africa, Asia and South America. While primary resistance to amoxicillin was at a low level in Europe (0\% to less than 2\%), it was higher in Africa, Asia and South America (6\% to $59 \%$ ). Similarly, resistance to tetracycline in most countries was either at a low level $(<5 \%)$ or had never existed, while it was higher in Asia and South America; 9\% to 27\% (34). These differences may be justified by the presence of different $H$. pylori strains, all over the world as well as different levels of antibiotic misuse (34). In conclusion, prevalence of $H$. pylori infection in our patients was $83 \%$, similar to that of developing countries. Moreover, severity of gastritis was correlated with the extent of $H$. pylori infection. In addition, the antibiotic resistance rate observed in this study signifies the ever-growing importance of further antibiotic sensitivity studies to help with proper treatment regimens against H. pylori.

\section{Acknowledgements}

The Effort made by Dr. Maziyar Rezvani and esteemed personer of Endoscopy and Gastroenterology Department of Shahid Beheshti University of Medical Sciences hospitals is appreciated.

\section{Authors' Contribution}

All authors collaborated in the preparation of this paper, specificallly Dr. Eslami, Mr. Shakeri, Mrs. Baseri and Mr. Montazeri.

\section{Financial Disclosure}

No conflicts of interest were declared.

\section{Funding/Support}

The study was financially supported by Shahid Beheshti University of Medical Sciences.

\section{References}

1. Marshall BJ, Warren JR. Unidentified curved Bacilli in the stomach of patients with gastritis and peptic ulceration. Lancet. 1984;323(8390):1311-1315.

2. Kersulyte D, Mukhopadhyay AK, Velapatino B, Su W, Pan Z, Garcia $\mathrm{C}$, et al. Differences in genotypes of Helicobacter pylori from different human populations. J Bacteriol. 2000;182(11):3210-8.

3. Cover TL, Blaser MJ. Helicobacter pylori in health and disease. Gastroenterology. 2009;136(6):1863-73.

4. Kusters JG, van Vliet AH, Kuipers EJ. Pathogenesis of Helicobacter pylori infection. Clin Microbiol Rev. 2006;19(3):449-90.

5. Tomasevic R, Golubovic G, Kiurski M, Stankovic D, Doder R, Pavlovic A. [Association of Helicobacter pylori infection with nodular antritis and follicular gastritis]. Vojnosanit Pregl. 2006;63(3):313-5.

6. Bosques-Padilla FJ, Tijerina-Menchaca R, Perez-Perez GI, FloresGutierrez JP, Garza-Gonzalez E. Comparison of Helicobacter pylori prevalence in symptomatic patients in northeastern Mexico with the rest of the country: its association with gastrointestinal disease. Arch Med Res. 2003;34(1):60-3.

7. Vale FF, Vitor JM. Transmission pathway of Helicobacter pylori: does food play a role in rural and urban areas? Int J Food Microbiol. 2010;138(1-2):1-12.

8. Mohammadi M, Doroud D, Mohajerani N, Massarrat S. Helicobacter pylori antibiotic resistance in Iran. World J Gastroenterol. 2005;11(38):6009-13.

9. Singh V, Mishra S, Maurya P, Rao G, Jain AK, Dixit VK, et al. Drug resistance pattern and clonality in H. pylori strains. J Infect Dev Ctries. 2009;3(2):130-6.

10. Eisig JN, Silva FM, Barbuti RC, Rodriguez TN, Malfertheiner P, Moraes Filho JP, et al. Efficacy of a 7-day course of furazolidone, levofloxacin, and lansoprazole after failed Helicobacter pylori eradication. BMC Gastroenterol. 2009;9:38.

11. Khatibian M, Ajvadi Y, Nasseri-Moghaddam S, Ebrahimi-Dariani $\mathrm{N}$, Vahedi $\mathrm{H}$, Zendehdel $\mathrm{N}$, et al. Furazolidone-based, metronidazole-based, or a combination regimen for eradication of Helicobacter pylori in peptic ulcer disease. Arch Iran Med. 2007;10(2):161-7.

12. Dixon MF, Genta RM, Yardley JH, Correa P. Classification and grading of gastritis. The updated Sydney System. International Workshop on the Histopathology of Gastritis, Houston 1994. Am J Surg Pathol. 1996;20(10):1161-81.

13. Oleastro M, Menard A, Santos A, Lamouliatte H, Monteiro L, Barthelemy P, et al. Real-time PCR assay for rapid and accurate detection of point mutations conferring resistance to clarithromycin in Helicobacter pylori.J Clin Microbiol. 2003;41(1):397-402.

14. Wayne PA. Performance standards for antimicrobial susceptibility testing. Ninth informational supplement NCCLS document M100S9. National Committee for Clinical Laboratory Standards. 2008.

15. Smith JG, Li W, Rosson RS. Prevalence, clinical and endoscopic predictors of Helicobacter pylori infection in an urban population. Conn Med. 2009;73(3):133-7.

16. Akcam M. Helicobacter pylori and micronutrients. Indian Pediatr 2010;47(2):119-26 
17. Pandeya N, Whiteman DC, Australian Cancer Study. Prevalence and determinants of Helicobacter pylori sero-positivity in the Australian adult community.J Gastroenterol Hepatol. 2011;26(8):1283-9.

18. Nouraie M, Latifi-Navid S, Rezvan H, Radmard AR, Maghsudlu M, Zaer-Rezaii H, et al. Childhood hygienic practice and family education status determine the prevalence of Helicobacter pylori infection in Iran. Helicobacter. 2009;14(1):40-6.

19. Sasidharan S, Ghayethry B, Ravichandran M, Latha LY, Lachumy SJ, Leng KM, et al. Prevalence of Helicobacter pylori infection among patients referred for endoscopy: Gender and ethnic differences in Kedah, Malaysia. Asian Pac J Trop Dis. 2012;2(1):55-59.

20. Paunio M, Hook-Nikanne J, Kosunen TU, Vainio U, Salaspuro M, Makinen J, et al. Association of alcohol consumption and Helicobacter pylori infection in young adulthood and early middle age among patients with gastric complaints. A case-control study on Finnish conscripts, officers and other military personnel. Eur J Epidemiol.1994;10(2):205-9.

21. Parkinson AJ, Gold BD, Bulkow L, Wainwright RB, Swaminathan B, Khanna B, et al. High prevalence of Helicobacter pylori in the Alaska native population and association with low serum ferritin levels in young adults. Clin Diagn Lab Immunol. 2000;7(6):885-8.

22. Everhart JE. Recent developments in the epidemiology of Helicobacter pylori. Gastroenterol Clin North Am. 2000;29(3):559-78.

23. Rauws EA, Langenberg W, Houthoff HJ, Zanen HC, Tytgat GN. Cam pylobacter pyloridis-associated chronic active antral gastritis. A prospective study of its prevalence and the effects of antibacterial and antiulcer treatment. Gastroenterology.1988;94(1):33-40.

24. Genta RM, Gurer IE, Graham DY, Krishnan B, Segura AM, Gutierrez $\mathrm{O}$, et al. Adherence of Helicobacter pylori to areas of incomplete intestinal metaplasia in the gastric mucosa. Gastroenterology. 1996;111(5):1206-11.

25. Bani-Hani KE, Hammouri SM. Prevalence of Helicobacter py- lori in Northern Jordan. Endoscopy based study. Saudi Med J. 2001;22(10):843-7.

26. Takeuchi K, Ohno Y, Tsuzuki Y, Ando T, Sekihara M, Hara T, et al. Helicobacter pylori infection and early gastric cancer. J Clin Gastroenterol. 2003;36(4):321-4.

27. Siavoshi F, Saniee P, Latifi-Navid S, Massarrat S, Sheykholeslami A Increase in resistance rates of $\mathrm{H}$. pylori isolates to metronidazole and tetracycline--comparison of three 3-year studies. Arch Iran Med. 2010;13(3):177-87.

28. Koletzko S, Richy F, Bontems P, Crone J, Kalach N, Monteiro ML, et al. Prospective multicentre study on antibiotic resistance of Helicobacter pylori strains obtained from children living in Europe. Gut. 2006;55(12):1711-6.

29. Glenwright HD, Sidaway DA. The use of metronidazole in the treatment of acute ulcerative gingivitis. Br Dent J.1966;121(4):174-7.

30. Rafeey M, Ghotaslou R, Nikvash S, Hafez AA. Primary resistance in Helicobacter pylori isolated in children from Iran.J Infect Chemother. 2007;13(5):291-5.

31. Savari M, Abdollahi H, Zahedi MJ, Moghadam SD, Abasi MHB [Antibiotic-resistance patterns of Helicobacter pylori isolates obtained from patients in Kerman (2009)]. J Kerman U Med Sci. 2011;18(1):73-82.

32. Kohanteb J, Bazargani A, Saberi-Firoozi M, Mobasser A. Antimicrobial susceptibility testing of Helicobacter pylori to selected agents by agar dilution method in Shiraz-Iran. Indian J Med Microbiol. 2007;25(4):374-7.

33. Siavashi F, Heydarian E, Pourkhajeh Ah, Merat Sh, Asl Soleymani $\mathrm{H}$, Khatibian M, et al. Susceptibility of various strains of Helicobacter pylori to selected agents. Arch Iran Med. 2000;3(2).

34. Boyanova L, Mitov I. Geographic map and evolution of primary Helicobacter pylori resistance to antibacterial agents. Expert Rev Anti Infect Ther. 2010;8(1):59-70. 